\title{
DISSIPATIVE ORDINARY DIFFERENTIAL OPERATORS OF EVEN ORDER
}

\author{
BY \\ A. OLUBUmmo
}

1. Introduction. We consider the dissipative differential system of the form

$$
\begin{aligned}
y_{t}=\left(T y_{k}\right)_{k} & +\left(S y_{k-1}\right)_{k-1}+\left(R y_{k-2}\right)_{k-2}+\cdots \\
& +\left(E y_{x x x}\right)_{x x x}+\left(D y_{x x}\right)_{x x}+\left(C y_{x}\right)_{x}+(B y)_{x}+A y
\end{aligned}
$$

for $t>0$ and $-\infty \leqq a<x<b \leqq \infty$. Here, $y=\left(\eta^{1}, \eta^{2}, \ldots, \eta^{m}\right)$ is a function of $x$ and $t$ with values in an $m$-dimensional complex Euclidean space $E^{m}$ and $T, S, R, \ldots$, $E, D, C, B, A$ are $m \times m$ matrix-valued functions of $x$ alone. The inner product in $E^{m}$ is defined as usual by

$$
(y, z)=\sum_{i=1}^{m} \eta^{i} \bar{z}^{i} \quad \text { with }|y|=[(y, y)]^{1 / 2} .
$$

The aim of this paper is to study the Cauchy problem for the system (1.1).

We shall make the following three assumptions:

(1.2) $B=B^{*}, C=-C^{*}, D=-D^{*}, \ldots, T=-T^{*}$;

(1.3) $\mathfrak{D} \equiv B_{x}+A+A^{*} \leqq 0, \quad a<x<b$;

(1.4) $T(x)$ is nonsingular for each $x \in(a, b)$; the elements of $T, T_{x}, T_{x x}, \ldots, T_{k-1}$; $S, S_{1}, S_{2}, \ldots, S_{k-2} ; \ldots, B$ are absolutely continuous on each compact subinterval of $(a, b)$. Also the elements of $T_{k}, S_{k-1}, \ldots, B_{x}$ and $A$ are square integrable on each compact subinterval of $(a, b)$.

In order to formulate the problem, we shall introduce some notational conventions. We set $F=(I-\mathfrak{D}) / 2$, where $I$ is the identity $m \times m$ matrix, and write $L_{2}(a, b ; F)$ for the space of all vector-valued measurable functions $y$ for which $\int_{a}^{b}(F y, y) d x<\infty$. With an inner product $\langle y, z\rangle_{1}$ in $L_{2}(a, b ; F)$ defined by $\langle y, z\rangle_{1}$ $=\int_{a}^{b}(F y, y) d x$ and a norm $\|y\|_{1}=\left[\langle y, y\rangle_{1}\right]^{1 / 2}, L_{2}(a, b ; F)$ becomes a Hilbert space which we shall sometimes denote by $H_{1} . L_{2}\left(a, b ; F^{-1}\right)$ and $L_{2}(a, b ; I)$ are defined in a similar way and will also be denoted by $H_{2}$ and $H_{0}$ respectively. With an inner product $\langle y, z\rangle_{12}$ in the product space $H_{12}=H_{1} \times H_{2}$ defined by $\langle y, z\rangle_{12}=\left\langle y^{1}, z^{1}\right\rangle_{1}$ $+\left\langle y^{2}, z^{2}\right\rangle_{2}, H_{12}$ is again a Hilbert space. If $y, z$ are functions on $(a, b)$ to $E^{m}$, we shall write $\langle y, z\rangle=\int_{a}^{b}(y, z) d x$.

We now define two transformations $L_{21}^{00}$ and $L_{21}^{1}$ as follows:

$$
\begin{aligned}
L_{21}^{00} y^{1} & =\left(T y_{k}^{1}\right)_{k}+\left(S y_{k-1}^{1}\right)_{k-1}+\cdots+\left(D y_{x x}^{1}\right)_{x x}+\left(C y_{x}^{1}\right)_{x}+\left(B y^{1}\right)_{x}+A y^{1}, \\
D\left(L_{21}^{00}\right) & =\left[y^{1}: y^{1} \in H_{1}, y^{1} \text { smooth with compact support in }(a, b)\right]
\end{aligned}
$$

Received by the editors October 26, 1966. 


$$
L_{21}^{1} y^{1}=\left(T y_{k}^{1}\right)_{k}+\left(S y_{k-1}^{1}\right)_{k-1}+\cdots+\left(D y_{x x}^{1}\right)_{x x}+\left(C y_{x}^{1}\right)_{x}+\left(B y^{1}\right)_{x}+A y^{1},
$$

(1.6) $D\left(L_{21}^{1}\right)=\left[y^{1}: y^{1} \in H_{1}, y^{1}, y_{x}^{1}, y_{x x}^{1}, \ldots, y_{2 k-1}^{1}\right.$ absolutely continuous,

$$
\left.\left(T y_{k}^{1}\right)_{k}+\left(S y_{k-1}^{1}\right)_{k-1}+\cdots+\left(B y^{1}\right)_{x}+A y^{1} \in H_{2}\right] .
$$

The closure of $L_{21}^{00}$ in the graph topology of $H_{1} \times H_{2}$ will be denoted by $L_{21}^{0}$, and the graph of $L_{21}^{00}$, i.e., the set of all pairs $\left\{\left[y^{1}, L_{21}^{00} y^{1}\right], y \in D\left(L_{21}^{00}\right)\right\}$ will be denoted by $G\left(L_{21}^{00}\right)$.

Suppose now that $y^{1}, z^{1} \in D\left(L_{21}^{1}\right)$; then integrating by parts successively and taking (1.2) into account, we obtain

$$
\begin{aligned}
\left\langle y^{1}, L_{21}^{1} z^{1}\right\rangle+\left\langle L_{21}^{1} y^{1}, z^{1}\right\rangle= & \left\langle y^{1},\left(T z_{k}^{1}\right)_{k}\right\rangle+\left\langle\left(T y_{k}^{1}\right)_{k}, z^{1}\right\rangle+\cdots \\
+ & \left\langle y^{1},\left(C z_{x}^{1}\right)_{x}\right\rangle+\left\langle\left(C y_{x}^{1}\right)_{x}, z^{1}\right\rangle+\left\langle y^{1},\left(B z^{1}\right)_{x}+A z^{1}\right\rangle \\
+ & \left\langle\left(B y^{1}\right)_{x}+A y^{1}, z^{1}\right\rangle \\
= & \int_{a}^{b}\left[\left(y^{1}, T z_{2 k-1}^{1}\right)_{x}+\left(T y_{2 k-1}^{1}, z^{1}\right)_{x}+\left(y^{1}, T_{x} z_{2 k-2}^{1}\right)_{x}\right. \\
& +\left(T_{x} y_{2 k-2}^{1}, z^{1}\right)_{x}+\cdots-\left(y_{x}^{1}, D z_{x x}^{1}\right)_{x} \\
& \left.-\left(D y_{x x}^{1}, z_{x}^{1}\right)_{x}+\left(y^{1}, C z_{x}^{1}\right)_{x}+\left(C y_{x}^{1}, z^{1}\right)_{x}+\left(B y^{1}, z^{1}\right)_{x}\right] d x \\
& +\left\langle\mathcal{D} y^{1}, z^{1}\right\rangle .
\end{aligned}
$$

Suppose that $y^{1}$ is a solution of (1.1) that is sufficiently smooth and let the differential operator on the right of (1.1) be denoted by $L y^{1}$. Then it follows from (1.7) that

$$
\left\langle y^{1}, y^{1}\right\rangle_{t}=\left\langle L y^{1}, y^{1}\right\rangle+\left\langle y^{1}, L y^{1}\right\rangle=-(\mathfrak{A} \bar{y}, \bar{y})^{a}+(\mathfrak{A} \bar{y}, \bar{y})^{b}+\left\langle\mathfrak{D} y^{1}, y^{1}\right\rangle,
$$

where

$$
\mathfrak{A}=\left(\begin{array}{lccccccc}
B & C & D_{x} & D+E_{x x} & . & . & . & T \\
C^{*} & 0 & -D & -E_{x} & . & . & . & 0 \\
D_{x}^{*} & -D^{*} & 0 & E & . & . & 0 & 0 \\
D^{*}+E_{x x}^{*} & . & . & . & . & . & 0 & 0 \\
. & . & T & . & . & . & . & . \\
. & -T^{*} & 0 & . & . & . & . & . \\
T^{*} & 0 & 0 & . & . & 0 & 0 & 0
\end{array}\right)
$$

and $\bar{y}=\left[y^{1}, y_{x}^{1}, y_{x x}^{1}, \ldots, y_{2 k-1}^{1}\right]$.

We note that the matrix $\mathfrak{A}$ is nonsingular since it is in triangular form and the diagonal terms, i.e., $T^{*},-T^{*}, \ldots, T$ are all nonsingular.

If in addition to the condition $\mathfrak{D} \leqq 0$, we require the solution to satisfy boundary conditions of the form

$$
-(\mathfrak{A} \bar{y}, \bar{y})^{a}+(\mathfrak{Q} \bar{y}, \bar{y})^{b} \leqq 0,
$$

then the solution "energy" will be nonincreasing in time. It is in this type of solution we are interested and accordingly, we shall impose the condition (1.8). Boundary conditions of this type will be called dissipative. The Cauchy problem for the 
system (1.1) can now be formulated in terms of semigroups of operators. We require of the operator

$$
L y=\left(T y_{k}\right)_{k}+\left(S y_{k-1}\right)_{k-1}+\cdots+\left(C y_{x}\right)_{x}+(B y)_{x}+A y
$$

with a domain $D(L)$ suitably restricted by dissipative boundary conditions that it generates a strongly continuous semigroup of bounded operators, say [S(t); $t \geqq 0]$. The initial value is here assumed in the mean square sense, i.e.,

$$
\text { St. } \lim _{t \rightarrow 0+} S(t) y_{0}=y_{0}, \quad y_{0} \in H_{0},
$$

and the differential equation is satisfied in the sense that the strong derivative

$$
(d S(t) / d t) y_{0}=L\left[S(t) y_{0}\right], \quad y_{0} \in D(L), t>0 .
$$

Our main object is to find all generators of this type. Furthermore we give in $\S 4$, a complete description of the boundary space associated with the system (1.1). The first- and second-order cases of this problem have been considered by Phillips [1] and Brooks [4], respectively, using a Green's function argument. In [5], Phillips and the present writer have considered the first- and second-order cases by a general operator-theoretic approach developed by Phillips in [2] and [3]. That it has been possible, in the general case treated in the present paper, to obtain all the information obtained in [1], [4], [5] by using the operator-theoretic approach is an indication of the power of that method.

The author wishes to express his gratitude to Professor Ralph Phillips for suggesting this more general investigation, for his inspiration, and for his helpful criticisms and encouragement in the preparation of this paper, and also to the referee for his suggestions.

2. Dissipative generators. We start this section by defining $2 k$ functions $g_{\varepsilon}^{(1)}(x, u), g_{\varepsilon}^{(2)}(x, u), \ldots, g_{\varepsilon}^{(2 k)}(x, u)$ as follows: Suppose that $j(x)$ is a function possessing derivatives of all orders and such that $j(x) \geqq 0, j(x)=0$ outside $[-1,1]$ and $\int j(x) d x=1$. Let $\varepsilon>0$ be fixed and let $x$ be a fixed number such that $a+\varepsilon<x<b$ $-\varepsilon$. We set $g_{\varepsilon}^{(1)}(x, u)=j_{\varepsilon}(x-u)$, where $j_{\varepsilon}(x)=(1 / \varepsilon) j(x / \varepsilon)$.

To define the remaining functions, let

$$
\begin{gathered}
a .<a^{\prime}<x<b^{\prime}<b, \quad \varepsilon<\varepsilon_{0}, \\
a+2 \varepsilon_{0}<a^{\prime}, \quad b-2 \varepsilon_{0}>b^{\prime}
\end{gathered}
$$

and write $a_{1}=a^{\prime}-\varepsilon_{0}$. We now take

$$
\begin{aligned}
& g_{\varepsilon}^{(2)}(x, u)=\int_{a}^{u}\left[j_{\varepsilon}(x-v)-j_{\varepsilon_{0}}\left(a_{1}-v\right)\right] d v ; \\
& g_{\varepsilon}^{(3)}(x, u)=\int_{a}^{u}\left[g_{\varepsilon}^{(2)}(x, v)-h_{3, \varepsilon}(x) j_{\varepsilon_{0}}\left(a_{1}-v\right)\right] d v, \\
& \text { where } h_{3, \varepsilon}(x)=\int_{a}^{b} g_{\varepsilon}^{(2)}(x, v) d v \text {; } \\
& g_{\varepsilon}^{(2 k)}(x, u)=\int_{a}^{u}\left[g_{\varepsilon}^{(2 k-1)}(x, v)-h_{2 k, \varepsilon}(x) j_{\varepsilon_{0}}\left(a_{1}-v\right)\right] d v, \\
& \text { where } h_{2 k, \varepsilon}=\int_{a}^{b} g_{\varepsilon}^{(2 k-1)}(x, v) d v \text {. }
\end{aligned}
$$


LEMMA 2.1. For each integer $r \geqq 3, h_{r, \varepsilon^{\prime}}(x)$ is bounded for all positive $\varepsilon^{\prime} \leqq \varepsilon$ and $\lim h_{r, \varepsilon}(x)$ exists as $\varepsilon \rightarrow 0$. For each integer $r>1, g_{\varepsilon}^{(r)}(x, u)$ is a smooth function vanishing together with all its derivatives near $a$ and $b$, and as $\varepsilon \rightarrow 0, g_{\varepsilon}^{(r)}(x, u)$ converges boundedly to a bounded measurable function of $u$.

Proof. The verification that $g_{\varepsilon}^{(2)}(x, u)$ is a smooth function vanishing together with all its derivatives near $a$ and $b$ is straightforward and is omitted. We proceed to prove the remaining two assertions of the lemma. First we note that $g_{\varepsilon}^{(2)}(x, u)$ has the stated properties. In fact, for all positive $\varepsilon^{\prime} \leqq \varepsilon$ and all $u$,

$$
\left|g_{\varepsilon^{\prime}}^{(2)}(x, u)\right| \leqq \int j_{\varepsilon_{0}}\left(a_{1}-u\right) d u .
$$

Also, as $\varepsilon \rightarrow 0$ and for all $u<x, g_{\varepsilon}^{(2)}(x, u)$ tends to a continuous function $g_{0}^{(2)}(x, u)$ which decreases from 0 near $u=a_{1}-\varepsilon_{0}$ and takes the constant value $\int j_{\varepsilon_{0}}\left(a_{1}-u\right) d u$ in $a^{\prime}<u<x$. For all $u>x, g_{0}^{(2)}(x, u)$ takes the constant value 0 . Hence $g_{0}^{(2)}(x, u)$ is a bounded measurable function of $u$ and $g_{\varepsilon}^{(2)}(x, u)$ has the stated properties. From this it also follows that $\lim _{\varepsilon \rightarrow 0} h_{3, \varepsilon}(x)=\lim _{\varepsilon \rightarrow 0} \int_{a}^{b} g_{\varepsilon}^{(2)}(x, v) d v=\int_{a}^{b} \lim _{\varepsilon \rightarrow 0} g_{\varepsilon}^{(2)}(x, v)$ exists. That $h_{3, \varepsilon^{\prime}}$ is bounded for all positive $\varepsilon^{\prime} \leqq \varepsilon$ is clear.

Next we consider $g_{\varepsilon}^{(3)}(x, u)$. Since $h_{3, \varepsilon^{\prime}}(x)$ is bounded for all positive $\varepsilon^{\prime} \leqq \varepsilon$ and $g_{\varepsilon^{\prime}}^{(2)}(x, u)$ is bounded for all positive $\varepsilon^{\prime} \leqq \varepsilon$ and all $u$ and vanishes near $a$ and $b$, $g_{\varepsilon^{\prime}}^{(3)}(x, u)$ is bounded for all $\varepsilon^{\prime} \leqq \varepsilon$ and all $u$. Further,

$$
\lim _{\varepsilon \rightarrow 0}\left\{g_{\varepsilon}^{(2)}(x, u)-h_{3, \varepsilon}(x) j_{\varepsilon_{0}}\left(a_{1}-u\right)\right\}
$$

exists as a bounded measurable function of $u$ and therefore,

$$
\begin{aligned}
\lim _{\varepsilon \rightarrow 0} g_{\varepsilon}^{(3)}(x, u) & =\lim _{\varepsilon \rightarrow 0} \int_{a}^{u}\left[g_{\varepsilon}^{(2)}(x, v)-h_{3, \varepsilon}(x) j_{\varepsilon_{0}}\left(a_{1}-v\right)\right] d v \\
& =\int_{a}^{u}\left[g_{0}^{(2)}(x, v)-h_{3,0}(x) j_{\varepsilon_{0}}\left(a_{1}-v\right)\right] d v
\end{aligned}
$$

exists and is a bounded and measurable function of $u$. Thus $g_{\varepsilon}^{(3)}(x, u)$ has the stated properties. Proceeding in this way, we have the result.

As in [5], we define a bilinear form $Q(y, z)$ on $H_{12}$ by setting

$$
Q(y, z)=\left\langle y^{1}, z^{2}\right\rangle+\left\langle y^{2}, z^{1}\right\rangle-\left\langle\mathscr{D} y^{1}, z^{1}\right\rangle,
$$

where $y=\left[y^{1}, y^{2}\right], z=\left[z^{1}, z^{2}\right]$ in $H_{12}$ and denote the $Q$-orthogonal complement of a set $S \subset H_{12}$ by $S^{\prime}$.

LEMMA 2.2. $G\left(L_{21}^{1}\right)=G\left(L_{21}^{00}\right)^{\prime}$.

Proof. We first note that $G\left(L_{21}^{1}\right) \subset G\left(L_{21}^{00}\right)^{\prime}$. In fact, let $z=\left[z^{1}, z^{2}\right] \in G\left(L_{21}^{1}\right)$; then we only need to show that $Q(y, z)=0$ for every $y=\left[y^{1}, L_{21}^{00} y^{1}\right]$ in $G\left(L_{21}^{00}\right)$. But this follows from (1.7) since under the hypothesis, $y^{1}$ vanishes at the end points $a$ and $b$. To prove that $G\left(L_{21}^{00}\right)^{\prime} \subset G\left(L_{21}^{1}\right)$, we suppose that $z \in G\left(L_{21}^{00}\right)^{\prime}$, i.e., $Q(y, z)=0$ for all $y \in G\left(L_{21}^{00}\right)$. Then if $z=\left[z^{1}, z^{2}\right]$, it has to be proved that $z^{1}, z_{x}^{1}, z_{x x}^{1}, \ldots, z_{2 k-1}^{1}$ are 
absolutely continuous, that $z^{1} \in L_{2}(a, b ; F)$ and that $z^{2}=L_{21}^{1} z^{1} \in L_{2}\left(a, b ; F^{-1}\right)$. We note that if $z^{1}, z_{x}^{1}, \ldots, z_{2 k-1}^{1}$ have all been shown to be absolutely continuous, then integration by parts gives

$$
\begin{aligned}
0 & =Q(y, z)=\left\langle y^{1}, z^{2}\right\rangle+\left\langle L_{21}^{00} y^{1}, z^{1}\right\rangle-\left\langle\mathscr{D} y^{1}, z^{1}\right\rangle \\
& =\left\langle y^{1}, z^{2}-\left(T z_{k}^{1}\right)_{k}-\left(S z_{k-1}\right)_{k-1}-\cdots-\left(B z^{1}\right)_{x}-A z^{1}\right\rangle
\end{aligned}
$$

for all smooth $y^{1}$ with compact support. It follows that $z^{2}=\left(T z_{k}^{1}\right)_{k}+\left(S z_{k-1}\right)_{k-1}$ $+\cdots+\left(B z^{1}\right)_{x}+A z^{1}$, and $z^{2} \in G\left(L_{21}^{1}\right)$.

To prove that $z$ is absolutely continuous, let $\phi$ be an arbitrary vector in $E^{m}$ and $g_{\varepsilon}^{(2 k)}(x, u)$ be as defined previously and substitute $y^{1}(u)=g_{\varepsilon}^{(2 k)}(x, u) \phi$ in

$$
0=Q(z, y)=\left\langle z^{1}, L_{21}^{1} y^{1}\right\rangle+\left\langle z^{2}, y^{1}\right\rangle-\left\langle\mathscr{D} z^{1}, y^{1}\right\rangle,
$$

i.e., in

$$
\begin{aligned}
0= & \left\langle z^{1}, T y_{2 k}^{1}+k T_{x} y_{2 k-1}^{1}+\cdots\right\rangle+\left\langle z^{1}, S y_{2 k-2}^{1}+(k-1) S_{x} y_{2 k-3}^{1}+\cdots\right\rangle \\
& +\cdots+\left\langle z^{1}, B y_{x}^{1}\right\rangle-\left\langle A z^{1}, y^{1}\right\rangle+\left\langle z^{2}, y^{1}\right\rangle .
\end{aligned}
$$

We then have

$$
\begin{aligned}
0=- & \int_{a}^{b}\left(\left(T z^{1}\right)(u),\left[-j_{\varepsilon}^{\prime}(x-u)+j_{\varepsilon_{0}}^{\prime}\left(a_{1}-u\right)-h_{3, \varepsilon}(x) j_{\varepsilon_{0}}^{\prime \prime}\left(a_{1}-u\right)+\cdots\right.\right. \\
& \left.\left.+h_{2 k, \varepsilon}(x) j_{\varepsilon_{0}}^{(2 k-1)}\left(a_{1}-u\right)\right] \phi\right) d u \\
-k \int_{a}^{b}\left(\left(T_{u} z^{1}\right)(u),\left[j_{\varepsilon}(x-u)-j_{\varepsilon_{0}}\left(a_{1}-u\right)+h_{3, \varepsilon}(x) j_{\varepsilon_{0}}^{\prime}\left(a_{1}-u\right)\right.\right. & \left.\left.+\cdots-h_{2 k, \varepsilon}(x) j_{\varepsilon_{0}}^{(2 k-2)}\left(a_{1}-u\right)\right] \phi\right) d u \\
& +\cdots+\cdots+\int_{a}^{b}\left(z^{2}(u)-\left(A z^{\prime}\right)(u), g_{\varepsilon}^{(2 k)}(x, u) \phi\right) d u .
\end{aligned}
$$

Since this is true for arbitrary $\phi \in E^{m}$, we have

$$
\begin{aligned}
\int_{a}^{b}\left(T z^{1}\right)(u) j_{\varepsilon}^{\prime}(x-u) d u-k \int_{a}^{b}\left(T_{u} z^{1}\right)(u) j_{\varepsilon}(x-u) d u \\
+\left\{\text { integrals of terms involving } g_{\varepsilon}^{(2 k)}(x, u), \ldots, g_{\varepsilon}^{(2)}(x, u)\right\}_{1}+K_{1}=0,
\end{aligned}
$$

where $K_{1}$ denotes the sum of terms involving $h_{2 k, \varepsilon}(x) j_{\varepsilon_{0}}^{\prime}\left(a_{1}-u\right)$,

$$
\begin{aligned}
h_{2 k, \varepsilon}(x) j_{\varepsilon_{0}}^{\prime \prime}\left(a_{1}-u\right), & \ldots, h_{2 k, \varepsilon}(x) j_{\varepsilon_{0}}^{(2 k-1)}\left(a_{1}-u\right) ; \quad h_{2 k-1, \varepsilon}(x) j_{\varepsilon_{0}}^{\prime}\left(a_{1}-u\right), \ldots, \\
& h_{2 k-1, \varepsilon}(x) j_{\varepsilon_{0}}^{(2 k-2)}\left(a_{1}-u\right) ; \ldots ; j_{\varepsilon_{0}}\left(a_{1}-u\right) \text { and } j_{\varepsilon_{0}}^{\prime}\left(a_{1}-u\right) .
\end{aligned}
$$

Writing $f_{\varepsilon}(x)=\int_{a}^{b} j_{\varepsilon}(x-u) f(u) d u$, we have

$$
\frac{\partial}{\partial x}\left(T z^{1}\right)_{\varepsilon}(x)=k\left(T_{u} z^{1}\right)_{\varepsilon}(x)-\{\}_{1}-K_{1},
$$

which we write as

$$
\left(T z^{1}\right)_{\varepsilon}^{\prime}=k\left(T_{u} z^{1}\right)_{\varepsilon}-\{\}_{1}-K_{1} .
$$

Now it can be shown as in Lemma 1.2.1 of [5] that $T z^{1}, T_{x} z^{1}, T_{x x} z^{1}, \ldots, T_{k} z^{1}$; $S z^{1}, S_{x} z^{1}, \ldots, S_{k-1} z^{1} ; \ldots ; B z^{1}, A z^{1}$ and $z^{2}$ all belong to $L_{1}\left(a^{\prime}, b^{\prime} ; I\right)$. Further, 
since by Lemma 2.1 each $g_{\varepsilon}^{(2)}(x, u)$ converges boundedly to a bounded measurable function of $u$, as $\varepsilon \rightarrow 0$, each of the terms

$$
\int_{a}^{b}\left(T_{u u} z^{1}\right)(u) g_{\varepsilon}^{(2)}(x, u) d u, \ldots, \int_{a}^{b}\left[z^{2}(u)-\left(A z^{1}\right)(u)\right] g_{\varepsilon}^{(2 k)}(x, u) d u
$$

will tend to a limit uniformly in $\left[a^{\prime}+2 \varepsilon_{0}, b^{\prime}-2 \varepsilon_{0}\right]$ and a fortiori in the $L_{1}$-norm. Thus $\left(T z^{1}\right)_{\varepsilon} \rightarrow \omega$, say, in $L_{1}\left[a^{\prime}+2 \varepsilon_{0}, b^{\prime}-2 \varepsilon_{0}\right]$ as $\varepsilon \rightarrow 0$. That $T z^{1}$ is absolutely continuous now follows exactly as in Lemma 1.2.1 of [5] and from this, it follows that $z^{1}$ is absolutely continuous.

Next, to prove that $z_{x}^{1}$ is absolutely continuous, we write the equation $0=Q(z, y)$ in the form

$$
\begin{aligned}
0= & \left\langle z^{1},\left(T y_{2 k-1}^{1}+(k-1) T_{x} y_{2 k-2}^{1}+\cdots+T_{k-1} y_{k}^{1}\right)_{x}\right\rangle \\
& +\left\langle z^{1},\left(S y_{2 k-3}+\cdots+S_{k-2} y_{k-1}^{1}\right)_{x}\right\rangle+\cdots-\left\langle A z^{1}, y^{1}\right\rangle+\left\langle z^{2}, y^{1}\right\rangle
\end{aligned}
$$

and integrate by parts, obtaining

$$
0=-\left\langle z_{x}^{1}, T y_{2 k-1}^{1}+(k-1) T_{x} y_{2 k-2}^{1}+\cdots+T_{k-1} y_{k}^{1}\right\rangle+\cdots+\left\langle z^{2}, y^{1}\right\rangle .
$$

We now set $y^{1}(u)=g_{\varepsilon}^{(2 k-1)}(x, u) \phi$. Then

$$
\begin{aligned}
0= & -\int_{a}^{b}\left(\left(T z_{u}^{1}\right)(u),\left[-j_{\varepsilon}^{\prime}(x-u)+j_{\varepsilon_{0}}^{\prime}\left(a_{1}-u\right)+\cdots+h_{2 k-1, \varepsilon}(x) j_{\varepsilon_{0}}^{(2 k-3)}\left(a_{1}-u\right)\right] \phi\right) d u \\
& +(k-1) \int_{a}^{b}\left(\left(T_{u} z_{u}^{1}\right)(u),\left[j_{\varepsilon}(x, u)-j_{\varepsilon_{0}}\left(a_{1}-u\right)-\cdots+h_{2 k-1}(x) j_{\varepsilon_{0}}^{(2 k-2)}\left(a_{1}-u\right)\right] \phi\right) d u \\
& +\cdots+\int_{a}^{b}\left(z^{2}(u), g_{\varepsilon}^{(2)}(x, u) \phi\right) d u
\end{aligned}
$$

whence

$$
\frac{\partial}{\partial x}\left(T z_{u}^{1}\right)_{\varepsilon}(x)=(k-1)\left(T_{u} z_{u}^{1}\right)_{\varepsilon}+\{\}_{2}+K_{2} .
$$

Generally, to prove $z_{r}^{1}$ is absolutely continuous, we write the equation $0=Q(z, y)$ in the form

$$
0=\left\langle z_{r}^{1}, T y_{2 k-r}^{1}\right\rangle+\left[\text { integrals of terms involving } y_{(2 k-r)-1}^{1}, y_{(2 k-r)-2}^{1}, \ldots, y^{1}\right] \text {. }
$$

We then set $y^{1}(u)=g_{\varepsilon}^{(2 k-r)}(x, u) \phi$ and argue as before. This concludes the proof.

COROLlary. $L_{21}^{0} \subset L_{21}^{1}$ and $D\left(L_{21}^{0}\right)=\left[y^{1} ; y^{1} \in D\left(L_{21}^{1}\right)\right.$ and $(\mathfrak{A} \bar{y}, \bar{z})_{a}^{b}=0$ for all $z^{1} \in D\left(L_{21}^{1}\right)$, where $\bar{y}=\left[y^{1}, y_{x}^{1}, \ldots, y_{2 k-1}^{1}\right]$ and $\left.\bar{z}=\left[z^{1}, z_{x}^{1}, \ldots, z_{2 k-1}^{1}\right]\right]$.

This corollary can be proved in a way analogous to the method used in proving the corollary to Lemma 1.2.1 of [5].

If $S_{0}$ and $S_{1}$ denote $G\left(L_{21}^{0}\right)$ and $G\left(L_{21}^{1}\right)$ respectively, then it is clear that $S_{0}$ is a null space, $S_{1}=S_{0}^{\prime}$ and that $S_{0} \subset S_{1}$. The quotient space $H=S_{1} / S_{0}$ is, in the terminology of [3], a boundary space. In the usual topology for a quotient space, $\boldsymbol{H}$ is a Hilbert space and $\boldsymbol{H}$ is isomorphic and isometric to $S_{1} \cap S_{0}^{\perp}$, the inner product in $S_{1} \cap S_{0}^{\perp}$ being the inner product $\langle y, z\rangle_{12}$ in $H_{12}$. Denoting the points of $\boldsymbol{H}$ by $\boldsymbol{y}, z, \ldots$, we shall express the fact that $y \in S_{1}$ belongs to the coset $y \in \boldsymbol{H}$ by writing 
$\bar{\beta} y=y$. We define a bilinear form $Q$ on $\boldsymbol{H}$ by setting $Q(\bar{\beta} y, \beta u)=Q(\beta y, \beta u)$; then it can be shown that $Q$ is a continuous, regular Hermitian bilinear form.

Definition. A transformation $T_{21}$ on $H_{1}$ to $H_{2}$ is said to engender the operator $T$ on $H_{0}$ to itself where $D(T)=\left[y^{1} ; y^{1} \in D\left(T_{21}\right)\right.$ and $\left.T_{21} y^{1} \in H_{0}\right]$, and $T y^{1}=T_{21} y^{1}$. Suppose now that $L^{0}$ and $L^{1}$ denote the operators on $H_{0}$ engendered by $L_{21}^{0}$ and $L_{21}^{1}$ respectively. Then we have the following result on applying Theorem 3.3 of [3]:

THEOREM 2.3. There is a one-to-one correspondence between the maximal negative subspaces $[N]$ of $\boldsymbol{H}$, taken with respect to $\boldsymbol{Q}$, and the maximal dissipative operators $[L]$ on $H_{0}$ such that $L^{0} \subset L \subset L^{1}$, the correspondence being defined by

which is dense in $H_{0}$.

$$
D(L)=\left[y^{1} ; \tilde{\beta}\left[y^{1}, L^{1} y^{1}\right] \in N\right],
$$

The following is Theorem 1.1.3 of [2]:

THEOREM 2.4. A necessary and sufficient condition for an operator $L$ to generate a strongly continuous semigroup of contraction operators on a Hilbert space $H$ to itself is that $L$ be a maximal dissipative operator with dense domain.

Combining Theorems 2.3 and 2.4 we obtain the desired solution to the Cauchy problem for the system (1.1).

3. Solutions of the homogeneous equations. Consider the system of ordinary differential equations

$$
\left(T y_{k}\right)_{k}+\left(S y_{k-1}\right)_{k-1}+\cdots+\left(C y_{x}\right)_{x}+(B y)_{x}+A y-\lambda y=0
$$

where $\lambda$ is a complex parameter and $x \in(a, b)$.

Let $\Gamma(x)$ be an $m \times m$ matrix-valued function of $x$ such that the elements of $\Gamma$ are measurable functions of $x$ and Lebesgue integrable on compact subintervals of $(a, b)$. Consider the ordinary first-order system of equations

$$
u_{x}(x)=\Gamma(x) u(x),
$$

where $u(x)$ is a vector-valued function of $x$ to $E^{m}$. For a proof of the following well-known lemma see, for example, Coddington and Levinson [6, p. 97, Problem 1].

LeMma 3.1. Let $\xi$ be an arbitrary vector in $E^{m}$ and let $c \in(a, b)$. There exists a unique vector-valued function $u$ of $x$ to $E^{m}$, which is absolutely continuous on compact subintervals of $(a, b)$, satisfies (3.2) for almost all $x \in(a, b)$, and is such that $u(c)=\xi$.

Set

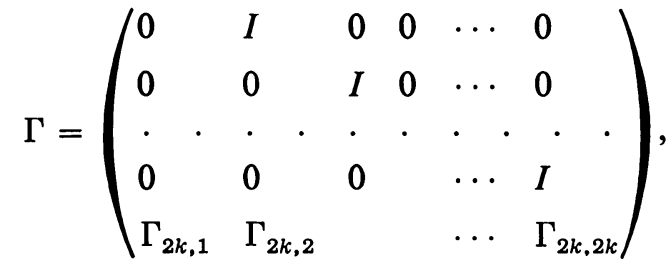

$$
\begin{aligned}
& \Gamma_{2 k, 1}=T^{-1}\left(\lambda-A-B_{x}\right) \text {, } \\
& \Gamma_{2 k, 2}=T^{-1}\left(-B-C_{x}\right) \text {, } \\
& \Gamma_{2 k, 3}=T^{-1}\left(-C-D_{x x}\right) \text {, etc. }
\end{aligned}
$$


With $\Gamma$ as defined, we now use Lemma 3.1 as in the proof of Theorem 2.1 of [4] to obtain the following

THEOREM 3.2. Let $c \in(a, b)$ and let $\eta^{1}, \eta^{2}, \ldots, \eta^{2 k}$ be vectors in $E^{m}$. Then there exists a unique vector-valued function $y$ of $x$ to $E^{m}$ such that $y, y_{x}, y_{x x}, \ldots, y_{2 k-1}$ are absolutely continuous with square integrable derivatives on compact subintervals of $(a, b), y$ satisfies (3.1) for almost all $x \in(a, b)$ and we have $y(c)=\eta^{1}, y_{x}(c)=\eta^{2}, \ldots$, $y_{n-1}(c)=\eta^{2 k}$.

We shall need the following lemma in the proof of our next theorem.

LEMMA 3.3. Let $y$ be a solution of $(3.1)$; then $(\mathfrak{Q} \bar{y}, \bar{y})(x)$ is an increasing function of $x$ on $(a, b)$. If $c \in(a, b)$, then $y \in L^{2}(c, b ; F)$ if and only if $(\mathfrak{A} \bar{y}, \bar{y})^{b}<+\infty$.

The proof of this is straightforward and is therefore omitted. The following is the second main result of this section.

TheOREM 3.4. Let $F_{b}\left[F_{a}\right]$ denote the collection of all solutions of equation (3.1) such that $(\mathfrak{A} \bar{y}, \bar{y})^{b}<+\infty\left[(\mathfrak{A} \bar{y}, \bar{y})^{a}>-\infty\right]$ and for each $x$, let $\mathfrak{A}(x)$ have $q$ negative and $p$ positive eigenvalues. Then $F_{b}\left[F_{a}\right]$ is a linear subspace of the solution space of (3.1) of dimension $l_{b} \geqq k m\left[l_{a} \geqq k m\right]$. If $C_{b}\left[C_{a}\right]$ denotes the collection of all solutions of (3.1) such that $(\mathfrak{A} \bar{y}, \bar{y})^{b} \leqq 0\left[(\mathfrak{A} \bar{y}, \bar{y})^{a} \geqq 0\right]$, then $C_{b} \subset F_{b}\left[C_{a} \subset F_{a}\right]$ and $C_{b}\left[C_{a}\right]$ contains at least one km-dimensional [km-dimensional] subspace.

Proof. An argument similar to that used in proving the corollary to Lemma 2.2 of [4] shows that $p=k m=q$. From Lemma 3.3, $F_{b} \subset L^{2}(c, b ; F)$. Further, $F_{b}$ is a linear subspace of $L^{2}(c, b ; F)$ since equation (3.1) is linear. Let $\left\{y_{1}, y_{2}, \ldots, y_{2 k m}\right\}$ be a basis for the solutions of (3.1); then if $y$ is an arbitrary solution of (3.1), there exist constants $\alpha_{i}$ such that $y=\sum_{i=1}^{2 k m} \alpha_{i} y^{i}$. Now $(\mathfrak{A} \bar{y}, \bar{y})^{x}=\sum_{i, j=1}^{2 k m} \alpha_{i} \bar{\alpha}_{j}\left(\mathfrak{A} \bar{x}_{i}, \bar{y}_{j}\right)^{x}$, and if we let $Y$ denote the $2 \mathrm{~km} \times 2 \mathrm{~km}$ matrix with its $i$ th column equal to $\bar{y}_{i}$, then the matrix of the above form is the nonsingular hermitian matrix $Y^{*}(x) \mathfrak{A}(x) Y(x)$ which again has $\mathrm{km}$ negative and $\mathrm{km}$ positive eigenvalues. Let

$$
C_{x}=\left[y:(\mathfrak{A} \bar{y}, \bar{y})^{x} \leqq 0\right] ;
$$

then $C_{x_{2}} \subset C_{x_{1}}$ if $x_{1} \leqq x_{2}$ and each $C_{x}$ contains at least one $k m$-dimensional subspace. Further, we have $C_{b}=\bigcap_{x \leqq b} C_{x}$.

Now the $k m$-dimensional subspaces are compact (in a suitable topology) and hence $C_{b}$ contains at least one $k m$-dimensional subspace. The results for the $a$ end of the interval are proved in a similar way. This concludes the proof.

4. Boundary behavior. Our aim in this section is to obtain more information about the boundary space $\boldsymbol{H}$. We first transform the system (1.1) into a new system

$$
\hat{L}_{21} y^{1}=\left(\hat{T} y_{k}^{1}\right)_{k}+\left(\hat{S} y_{k-1}^{1}\right)_{k-1}+\cdots+\left(\hat{C} y_{x}^{1}\right)_{x}+\left(\hat{B} y^{1}\right)_{x}+\hat{f} y^{1}
$$

so that $\hat{\mathfrak{D}}=0$. For this purpose we set $\hat{T}=T, \hat{S}=S, \ldots, \hat{C}=C, \hat{B}=B, \hat{A}=\left(A-A^{*}\right) / 2$ $-B_{x} / 2$; then $\hat{\mathfrak{D}}=0$ and $A-\hat{A}=\mathfrak{D} / 2$. 
As in $\S 3$ of [5], we define an operator $\sigma$ on $H_{12}$ to itself by

$$
\sigma\left[y^{1}, y^{2}\right]=\left[y^{1}, y^{2}+(\hat{A}-A) y^{1}\right]
$$

then $\sigma$ is regular and if we define $\hat{Q}$ by $\hat{Q}(y, z)=\left\langle y^{1}, z^{2}\right\rangle+\left\langle y^{2}, z^{1}\right\rangle$, we have

$$
\hat{Q}(\sigma y, \sigma z)=Q(y, z) \text {. }
$$

Further, setting $\hat{S}_{1}=\sigma S_{1}, \hat{S}_{0}=\sigma S_{0}, \boldsymbol{H}=S_{1} / S_{0}$ and $\hat{\boldsymbol{H}}=\hat{S}_{1} / \hat{S}_{0}$, it is easily verified that $\sigma$ induces a one-to-one bicontinuous mapping of $\boldsymbol{H}$ onto $\hat{\boldsymbol{H}}$ taking maximal negative (maximal positive) subspaces of $\boldsymbol{H}$ into maximal negative (maximal positive) subspaces of $\hat{\boldsymbol{H}}$. Moreover, arguing precisely as in $\$ 1.3$ of [5], we see that there is a one-to-one correspondence between the boundary space $\hat{\boldsymbol{H}}$ (and therefore the boundary space $\boldsymbol{H}$ ) and the set of all solutions of the equation

$$
\left(F^{-1} \hat{L}_{21}^{1}-I\right)\left(F^{-1} \hat{L}_{21}^{1}+I\right) y^{1}=0 .
$$

In order to determine the set of solutions of (4.2), we consider the two equations

$$
\left(F^{-1} \hat{L}_{21}^{1}-I\right) y^{1}=0
$$

and

$$
\left(F^{-1} \hat{L}_{21}^{1}+I\right) y^{1}=0
$$

that is

$$
\left(T y_{k}^{1}\right)_{k}+\left(S y_{k-1}^{1}\right)_{k-1}+\cdots+\left(C y_{x}^{1}\right)_{x}+\left(B y^{1}\right)_{x}+A y^{1}-y^{1} / 2=0
$$

and

$$
-\left(T y_{k}^{1}\right)_{k}-\left(S y_{k-1}^{1}\right)_{k-1} \cdots-\left(C y_{x}^{1}\right)_{x}-\left(B y^{1}\right)_{x}-B_{x} y^{1}+A^{*} y^{1}-y^{1} / 2=0 .
$$

As in Theorem 3.4, $\mathfrak{A}$ has $\mathrm{km}$ negative and $\mathrm{km}$ positive eigenvalues. Since $\mathfrak{A}$ is a nonsingular hermitian matrix, it is clear that $p+q=2 \mathrm{~km}$. Using Theorem 3.4 , we now have

THEOREM 4.1. If $F_{b}\left[F_{a}\right]$ denotes the collection of all solutions of (4.3) such that $(\mathfrak{U} \bar{y}, \bar{y})^{b}<+\infty\left[(\mathfrak{H} \bar{y}, \bar{y})^{a}>-\infty\right]$, then $F_{b}\left[F_{a}\right]$ is a linear subspace of the solution space of (4.3), of dimension say, $l_{b} \geqq k m\left[l_{a} \geqq k m\right]$. If $C_{b}\left[C_{a}\right]$ denotes the collection of all solutions of (4.3) such that $(\mathfrak{A} \bar{y}, \bar{y})^{b} \leqq 0\left[(\mathfrak{H} \bar{y}, \bar{y})^{a} \geqq 0\right]$, then $C_{b} \subset F_{b}\left[C_{a} \subset F_{a}\right]$ and $C_{b}\left[C_{a}\right]$ contains at least one km-dimensional [km-dimensional] subspace.

A similar result holds for the equation (4.4). The set $G_{b}\left[G_{a}\right]$ of solutions $z^{1}$ such that $(\mathfrak{U} \bar{z}, \bar{z})^{b}>-\infty\left[(\mathfrak{U} \bar{z}, \bar{z})^{a}<+\infty\right]$ is of dimension say, $m_{b} \geqq k m\left[m_{a} \geqq k m\right]$ and the set for which $(\mathfrak{A} \bar{z}, \bar{z})^{b} \geqq 0\left[(\mathfrak{A} \bar{z}, \bar{z})^{a} \leqq 0\right]$ contains a $k m$-dimensional [ $k m$-dimensional] subspace.

Arguing now as in $\$ 1.3$ of [5], we construct a special basis for the set of all square integrable solutions of (4.3). Let $N_{a}$ and $N_{b}$ be a fixed $\mathrm{km}$-dimensional and a fixed $\mathrm{km}$-dimensional subspace contained in $C_{a}$ and $C_{b}$, respectively. Then it is easy to see that $N_{a}$ and $N_{b}$ are linearly independent. Thus $N_{a}$ and $N_{b}$ together span the 
$2 k m$-dimensional solution space of (4.3). We can therefore choose $l_{b}-k m y_{a}^{1}$, s in $N_{a}$ which together with $N_{b}$ span $F_{b}$. These $l_{b}-k m$ functions will be square integrable at both ends $a$ and $b$. Similarly, we have $l_{a}-k m$ functions from $N_{b}$ which are square integrable at both ends, i.e., which belong to $L_{2}(a, b ; F)$. Moreover, these $\left(l_{b}-k m\right)+\left(l_{a}-k m\right)$ functions span the space of all solutions which are square integrable at both ends. Also the space of solutions of (4.4) which are square integrable at both ends, i.e., which belong to $L_{2}(a, b ; F)$ is of dimension $\left(m_{a}-k m\right)$ $+\left(m_{b}-k m\right)$, and again it can be shown as in $\$ 1.3$ of [5] that the square integrable solutions of (4.3) and (4.4) comprise all of the solutions of equation

$$
\left(F^{-1} \hat{L}_{22}^{1}-I\right)\left(F^{-1} \hat{L}_{21}^{1}+I\right) y^{1}=0 .
$$

Finally, we note that if $y=\left[y^{1}, L_{21}^{1} y^{1}\right]$ and $y^{1}$ is a solution of (4.3), then $Q(y, y)>0$ and if $z=\left[z^{1}, L_{21}^{1} z^{1}\right]$ and $z^{1}$ is a solution of (4.4), then $Q(z, z)<0$.

Since as we have seen, there is a one-to-one correspondence between the boundary space $H$ and the set of all solutions of the equations (4.2) we have the following:

THEOREM 4.2. Let $d_{H}, d_{N}, d_{P}$ denote the dimensions of the boundary space, the negative subspace of $Q$ and the positive subspace of $Q$ respectively. Then

$$
d_{H}=l_{a}+l_{b}+m_{a}+m_{b}-4 k m, \quad d_{N}=m_{a}+m_{b}-2 k m,
$$

and

$$
d_{P}=l_{a}+l_{b}-2 k m \text {. }
$$

\section{REFERENCES}

1. R. S. Phillips, Dissipative hyperbolic systems, Trans. Amer. Math. Soc. 86 (1957), 109-173.

2. - Dissipative operators and hyperbolic systems of partial differential equations, Trans. Amer. Math. Soc. 90 (1959), 193-254.

3. - Dissipative operators and parabolic differential equations, Comm. Pure Appl. Math. 12 (1959), 249-276.

4. J. Brooks, Second order dissipative systems, Ph.D. Dissertation, Univ. of Southern California, Los Angeles, 1960.

5. A. Olubummo and R. S. Phillips, Dissipative ordinary differential operators, J. Math. Mech. 14 (1965), 929-950.

6. E. A. Coddington and N. Levinson, Theory of ordinary differential equations, McGrawHill, New York, 1955.

UNIVERSITY OF IBADAN, IBADAN, Nigeria 